\title{
MIMO channel capacity using antenna selection and water pouring
}

\author{
Jose V Cuan-Cortes, Cesar Vargas-Rosales ${ }^{*}$ and David Munoz-Rodriguez
}

\begin{abstract}
The use of multiple-input multiple-output (MIMO) communication systems has attracted considerable attention due to capacity and performance improvements without increasing the required bandwidth or transmission power. Although MIMO improves the transmission rate and provides reliable communication, these advantages come at a high cost, since multiple radio frequency (RF) chains have to be employed. Therefore, cost-effective implementation of MIMO systems remains as an important challenge. In this sense, antenna selection techniques (AST) can help reduce the high cost of MIMO systems, while retaining most of their benefits; and in order to improve channel capacity, transmission power can be allocated efficiently according to the channel experienced by each antenna element. In this paper, we show the channel capacity improvement of MIMO systems, by combining the use of AST and transmission power allocation through the water-pouring algorithm (WPA) for different antenna configurations. We apply WPA and allocate more power to the channel with better conditions, when the MIMO channel is Rayleigh distributed and noise is Gaussian. By employing these two techniques (AST and WPA), we show that channel capacity is significantly improved, with higher capacity values than those obtained using traditional systems which just spread equal power among all the transmit antennas. We also show that bounds on the capacity can be considered when these improvements are used in a MIMO system.
\end{abstract}

Keywords: MIMO; Ergodic capacity; Antenna selection technique; Water-pouring algorithm

\section{Introduction}

Communication systems that use MIMO form a key technology for next generation wireless systems, because they provide a reliable communication (using diversity techniques) or higher data rates (using spatial multiplexing techniques) that are enablers to achieve a channel capacity enhancement, without the need of using additional transmit power or bandwidth, [1-3]. MIMO uses antenna arrays instead of single transmit, receive antennas, and operates by simultaneously transmitting multiple signals in the same frequency band. At the receiver, multiple antennas are also used, and the received signals are processed to separate different transmitted data streams. These transmit-receive antenna pairs form wireless links that have different impulse responses, and thus channel conditions might be different for each one of them. Therefore, a MIMO system can use more efficiently these

*Correspondence: cvargas@itesm.mx

Department of Electrical and Computer Engineering, Tecnologico de Monterrey, Campus Monterrey, Ave. Eugenio Garza Sada 2501, Monterrey, NL 64849, Mexico resources by performing antenna selection in order to transmit through the best wireless links, and by allocating the necessary power to those antennas being chosen in order to achieve performance criteria. Antenna selection techniques choose a subset of the available transmission antennas by comparing the channels they find at the moment of transmission, so that performance criteria such as capacity, bit error rate (BER) or throughput are satisfied. In [4], authors present an overview of classic results on selection diversity and antenna selection algorithms at the transmit and receive side in MIMO systems. In [5], antenna selection with imperfect channel estimation is analyzed, and the optimal single receiver antenna selection rule is obtained. It is also shown that the number of receive antennas determines the complexity of the technique. In [6], antenna selection is also considered for MIMO-OFDM systems, although the selection is limited to a single antenna and no further consideration of extension to multiple antennas is made. It is known that antenna selection improves BER, but it is also known that performance improvement can be achieved if transmit

\section{望 Springer}

(c) 2014 Cuan-Cortes et al:- licensee Springer. This is an Open Access article distributed under the terms of the Creative Commons Attribution License (http://creativecommons.org/licenses/by/2.0), which permits unrestricted use, distribution, and reproduction in any medium, provided the original work is properly credited. 
power is varied according to channel conditions. In [7], building on the classical water-pouring algorithm, authors proposed an antenna selection algorithm based on partial water pouring over the strongest channel model but using equal power allocation. In [8], power control in MIMO systems is introduced with antenna selection using truncated channel inversion. In [9], antenna selection is shown for spatial multiplexing and diversity techniques for linear receivers. One of the techniques that were proposed is a suboptimal solution using the eigenstructure of the channel matrix to choose the number of antenna elements needed for an improved performance, no power allocation is carried out. Water-pouring algorithm (WPA) has also been used for antenna selection in [10], but it is not used for power allocation. In [11], authors used antenna selection in a long-term evolution (LTE), system primarily to quantify performance (symbol error rate and cumulative distribution function) and to provide a systematic overview of all the hooks in the LTE standard that enable transmit antenna selection. Recently, in [12], authors used antenna selection and power allocation to improve performance in massive MIMO systems. They use convex optimization to select the antenna subset that maximizes the dirty-paper coding capacity and then optimize over the user power allocation.

In this paper, we present a system that uses both, antenna selection and power allocation, to improve capacity. It is known that the channel capacity in a MIMO communication system depends on the number of transmit and received antennas, the signal-to-noise ratio, the channel state, and the autocorrelation or covariance matrix of the transmitted signal vector [1]. This covariance matrix is the variable that is left to improve channel capacity. We propose the improvement of the channel capacity through the use of a mathematical model which considers antenna selection techniques (AST) as well as the variation of the covariance matrix using WPA by assigning weights according to the channel coefficients of the channel matrix. We assume that the channel matrix and its coefficients are known or estimated previously. The weights obtained are used to determine the amount of power needed to be allocated to each antenna element of the subset that has been chosen for transmission. The technique to obtain the weights is based on the eigenvalues of the covariance matrix in an optimal way, thus improving performance over that reported in [9] and [10]. Since the covariance matrix is analyzed at the transmitter side, in this work, we are going to consider AST only at the transmitter.

In the 'MIMO communication systems' section, we present the basic notation with the definitions used in the analysis. The section 'MIMO system using I-AST and WPA' introduces the model proposed using antenna selection and WPA. The 'Numerical results' section are discussed afterwards for different antenna arrays, and a discussion on capacity bounds is introduced. At the end, the 'Conclusions' section is presented.

\section{MIMO communication systems}

A typical MIMO system consists of $M_{T}$ transmit and $M_{R}$ receive antennas. Since each pair of transmit-receive antennas experience different channel behaviors, the wireless MIMO channel is represented by using a channel matrix $\mathbf{H}$, whose dimensions are $M_{R} \times M_{T}$. The matrix element $h_{i, j}, i=1,2, \ldots, M_{R} ; j=1,2, \ldots, M_{T}$ is the channel's impulse response coefficient between the $i$-th receiving and $j$-th transmitting antennas. If we consider a transmitted symbol vector $\mathbf{x}$, which is composed of $M_{T}$ independent input symbols, i.e., $\mathbf{x}^{T}=\left[x_{1}, x_{2}, \ldots, x_{M_{T}}\right]$, the input-output relationship of the MIMO channel just described, in matrix form, gives for the received signal vector $\mathbf{y}$ in the following:

$$
\mathbf{y}=\sqrt{\frac{E_{s}}{M_{T}}} \mathbf{H x}+\mathbf{n},
$$

where $\mathbf{y}$ is an $M_{R} \times 1$ received signal vector, $E_{s}$ is the total average energy of the transmitted signal, and $\mathbf{n}$ is the noise vector with dimension $M_{R} \times 1$, considered to be Gaussian. The channel matrix $\mathbf{H}$ is a matrix with rank $r$ and with positive eigenvalues of $\mathbf{H H}^{H}$ generally denoted by $\lambda_{k}, k=$ $1,2, \ldots, r$.

\section{Antenna selection techniques}

It is known that the main advantage of MIMO systems is a substantial improvement in data rate and reliability, but its main drawback is that additional RF modules are required as multiple antennas are employed. In order to reduce the cost associated with the multiple RF modules, we can employ AST, where a specific number of $P$ antennas is selected at the transmitter or receiver [1,13], $\left(P \leq M_{T}\right.$ for selection at the transmitter and $P \leq M_{R}$ for selection at the receiver). We contemplate the antenna selection only at the transmitter side to optimize performance. The optimal algorithm involves an exhaustive search through all the possible combinations of $P$ antennas out of a total of $M_{T}$. That is, if we want to select three antennas $(P=3)$ from a set of four available antennas $\left(M_{T}=4\right)$, we calculate the channel capacity of all possible combinations of three antennas $\left(\begin{array}{c}M_{T} \\ P\end{array}\right)$; in this case, it would be four different possible combinations. The selection of the $P$ antennas affects the channel capacity equation in an iterative algorithm, which evaluates all possible antenna combinations to get the highest channel capacity, see [14]. This iterative search may be of high complexity, especially when the number of antennas is large. Some algorithms that avoid serial searches and with optimal performance have been proposed, we call those the incremental selection and the 
decremental selection. The incremental selection AST (IAST) adds successively antennas at every stage, so the antenna that yields the maximum increase of the channel capacity is added to the set of $P$ transmit antennas [14]. In other words, the antenna that provides the highest capacity is selected at first, then the highest second, and so on. The process continues until all $P$ antennas are selected. The decremental selection AST (D-AST) starts with the whole set of $M_{T}$ transmit antennas and removes one antenna in each of the $\left(M_{T}-P\right)$ stages, where the algorithm identifies and removes the antenna that yields the minimum contribution to capacity [14].

\section{Power allocation and water-pouring algorithm}

When the channel is unknown to the transmitter, assigning equal power to all transmit antenna is the logical solution to set up the MIMO communication link. If the channel is completely known, to assign power in an efficient way in agreement with the channel conditions will improve capacity. WPA is an iterative technique that efficiently allocates different levels of power to various transmitting antennas. The algorithm allocates more power to the antennas that experience channels that are in better conditions, and less or none at all, to the antennas whose channels are in bad conditions [3]. WPA has to satisfy the optimal power allocation policy, which is determined by:

$$
\gamma_{i}^{\mathrm{opt}}=\left(\mu-\frac{M_{T} N_{0}}{E_{s} \lambda_{i}}\right)_{+} ; i=1,2, \ldots, r,
$$

and

$$
\sum_{i=1}^{r} \gamma_{i}^{\mathrm{opt}}=M_{T}
$$

where $\gamma_{i}^{\text {opt }}$ is the optimum value of power to be allocated to transmitting antenna $i, \mu$ is a constant, $N_{0}$ is the noise power, and $(x)_{+}$is defined as:

$$
(x)_{+}=\left\{\begin{array}{l}
x, \text { if } x \geq 0 \\
0, \text { if } x<0
\end{array}\right.
$$

The WPA starts setting the iteration count index $p$ to 1 , and then, calculating the constant $\mu$ using:

$$
\mu=\frac{M_{T}}{(r-p+1)}\left[1+\frac{N_{0}}{E_{s}} \sum_{i=1}^{r-p+1} \frac{1}{\lambda_{i}}\right]
$$

Using this value of $\mu$, and using the definition in Equation 4, the power allocated to the $i$-th sub-channel can be obtained as:

$$
\gamma_{i}=\left(\mu-\frac{M_{T} N_{0}}{E_{s} \lambda i}\right)_{+}, i=1,2, \ldots, r-p+1 .
$$

As it can be seen in (6), if the energy allocated to the channel with the lowest gain is negative, i.e., $\gamma_{r-p+1}<0$, we discard this channel by setting $\gamma_{r-p+1}^{\text {opt }}=0$, which means that zero power is assigned to this channel, and then, the algorithm returns to the iteration and $p$ is incremented by 1. The optimal power allocation strategy, using WPA, is found when the power allocated to each spatial sub channel is non-negative [15]. Figure 1 illustrates the outcome of the WPA, where it can be seen that the modes obtained by (6) that are positive will allow the allocation of power to the $P$ antennas selected. Those bars in the figure that do not have a mode (those on the right hand side of the figure with no $\gamma_{i}$ ) belong to those antennas that were not allocated power.

Since this algorithm only concentrates on good-quality channels and discards the bad ones during each channel realization, it is to be expected that this method yields a capacity that is equal or better than the capacity when the channel is unknown to the transmitter [3]. This has been shown to be the improvement with different algorithms that carry out antenna selection, see [5-10].

\section{MIMO system using I-AST and WPA}

Once defined the concept and the application of I-AST and WPA over the MIMO system, we can develop the proposed model. Our proposal is to use these two techniques, since both improve the channel capacity and we can get a more cost-efficient MIMO system at the same time. As we saw in the antenna selection technique section, we need to choose the best set of $P$ antennas according to its channel coefficients using I-AST. Once those $P$ antennas are obtained, we apply the WPA over the resulting model. We suggest to apply I-AST first, because WPA is not able to select the best set of transmit antennas by itself. First, consider the input-output relationship of the MIMO system using I-AST, which is defined as:

$$
\mathbf{y}=\sqrt{\frac{E_{s}}{P}} \mathbf{H}_{K} \mathbf{x}_{P}+\mathbf{n}
$$

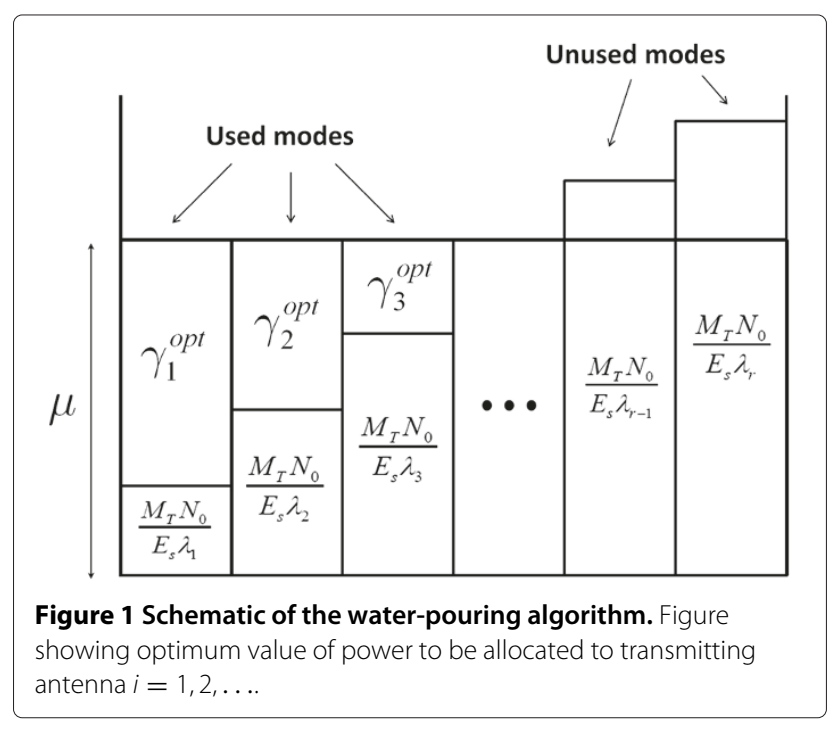


where $\mathbf{H}_{K}$ is the channel matrix indexed through the iterative process that looks for the set of $P$ antennas, and has size $\left(M_{R} \times P\right)$. The vector $\mathbf{x}_{P}$, is composed of $P$ independent input symbols, i.e., $\mathbf{x}_{P}^{T}=\left[x_{1}, x_{2}, \ldots, x_{P}\right]$.

Now, with knowledge of the channel state information (CSI), the system can pre-process the signal to be transmitted by certain linear transformation $\mathbf{V}$, and at the receiver, the signal can be post-processed by another linear transformation, $\mathbf{U}^{H},[1]$, as Figure 2 shows. $\mathbf{V}$ and $\mathbf{U}$ are matrices derived from the singular value decomposition (SVD) of the channel matrix $\mathbf{H}_{K}$. That is, if we apply SVD to the channel matrix $\mathbf{H}_{K}$, we obtain:

$$
\mathbf{H}_{K}=\mathbf{U} \Sigma \mathbf{V}^{H}
$$

where $\mathbf{U}$ and $\mathbf{V}$ are unitary matrices that satisfy $\mathbf{U}^{H} \mathbf{U}=$ $\mathbf{V}^{H} \mathbf{V}=\mathbf{I}$, and $\Sigma$ is a square matrix, whose diagonal elements are the singular values of the matrix $\mathbf{H}_{K}$ and whose off-diagonal elements are zero.

From the modal decomposition shown in Figure 2, the input-output relationship using WPA, is defined as:

$$
\widetilde{\mathbf{y}}=\sqrt{\frac{E_{s}}{M_{T}}} \mathbf{U}^{H} \mathbf{H} \mathbf{V} \widetilde{\mathbf{x}}+\widetilde{\mathbf{n}} .
$$

where $\widetilde{\mathbf{y}}=\mathbf{U}^{H} \mathbf{y}$ is a post-processed received signal vector; $\widetilde{\mathbf{x}}=\mathbf{V}^{H} \mathbf{x}$ is a pre-processed transmitted signal vector; and $\widetilde{\mathbf{n}}=\mathbf{U}^{H} \mathbf{n}$ is a post-processed noise vector.

Now, replacing $\mathbf{H}$ by $\mathbf{H}_{K}$ (channel matrix of the $P$ antennas selected), $\widetilde{\mathbf{x}}$ by $\widetilde{\mathbf{x}}_{P}$ (pre-processed signal vector), and $M_{T}$ by $P$ on Equation 9, we get the input-output relationship for the proposed model, where I-AST was applied first, and then WPA. Therefore, the new model is defined by:

$$
\widetilde{\mathbf{y}}=\sqrt{\frac{E_{s}}{P}} \mathbf{U}^{H} \mathbf{H}_{K} \mathbf{V} \widetilde{\mathbf{x}}_{P}+\widetilde{\mathbf{n}}
$$

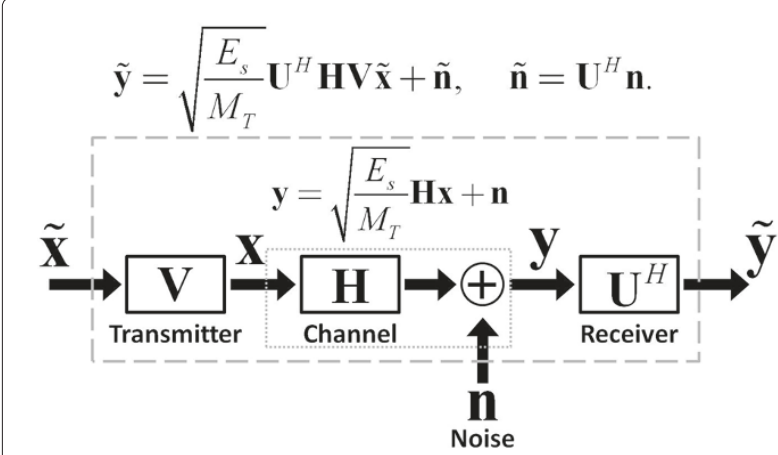

Figure 2 Model decomposition with CSI at transmitter and receiver. Figure showing block diagram of transmitter, channel, and receiver.
Now, we obtain an expression for the channel capacity of the proposed model. The channel capacity using I-AST is defined by:

$$
C=\max _{K, \mathbf{R}_{\overline{\mathbf{x x}}}} \log _{2}\left(\left|\mathbf{I}_{M_{R}}+\frac{E_{s}}{P N_{0}} \mathbf{H}_{K} \mathbf{R}_{\overline{\mathbf{x}} \mathbf{x}} \mathbf{H}_{K}^{H}\right|\right),
$$

where $|\mathbf{X}|$ is the determinant of matrix $\mathbf{X}$, the covariance matrix $\mathbf{R}_{\overline{\mathbf{X x}}}$ must be determined to satisfy the transmitter power constraints. Now, $\mathbf{R}_{\overline{\mathbf{x x}}}$ is obtained using WPA to get an optimum power allocation that satisfies the transmitter power condition $\left(\operatorname{Tr}\left(\mathbf{R}_{\overline{\mathbf{x x}}}\right)=M_{T}\right)$. Considering that optimal power allocation, across all spatial sub-channels, is determined by WPA, i.e., all $\gamma_{i}^{\text {opt }}$ are set in an optimum way, we can get the new expression for the covariance matrix $\mathbf{R}_{\overline{\mathbf{x x}}}$ [1], which is given by:

$$
\mathbf{R}_{\overline{\mathbf{X X}}}^{\mathrm{imp}}=\mathbf{V} \mathbf{R}_{\overline{\mathbf{X x}}}^{\mathrm{opt}} \mathbf{V}^{H},
$$

where $\mathbf{R}_{\overline{\mathbf{x x}}}^{\mathrm{opt}}=\operatorname{diag}\left\{\gamma_{1}^{\mathrm{opt}}, \gamma_{2}^{\mathrm{opt}}, \ldots, \gamma_{P}^{\mathrm{opt}}\right\}$ is the optimal covariance matrix and $\mathbf{V}^{H} \mathbf{V}=\mathbf{I}_{P}$. Therefore, the improved channel capacity according to the proposed model is given by:

$$
C_{\text {imp }}=\max _{K} \log _{2}\left(\left|\mathbf{I}_{M_{R}}+\frac{E_{s}}{P N_{0}} \mathbf{H}_{K} \mathbf{R}_{\overline{\mathbf{x x}}}^{\mathrm{imp}} \mathbf{H}_{K}^{H}\right|\right) .
$$

Finally, the ergodic case for Equation 13 is defined by using statistical averages of the random channel coefficients of matrix $\mathbf{H}_{K}$ with the expectation operator $\varepsilon$ as follows:

$$
\bar{C}_{\text {imp }}=\varepsilon\left[C_{\text {imp }}\right] .
$$

Equations 13 and 14 represent the channel capacity of our proposed model, where the main improvement is the use of the I-AST and WPA at the same time. With this model, we show in the following section that channel capacity improves. We also analyze the channel capacity for the models obtained and compare these capacities to know which model improves channel capacity.

\section{Numerical results}

A particular antenna array will be represented by the number of receive, $M_{R}$, and transmit, $M_{T}$, antennas as $M_{R} \times M_{T}$. We define an array of $4 \times 4$ as the basic scenario of the architecture of full rank. Since we are interested in working with antenna selection just at the transmitter side, the other arrays which we are going to treat will be represented by $4 \times 3,4 \times 2$, and $4 \times 1$ arrays and we call these antenna configurations. For our simulation, we consider a random channel, which will be chosen accordingly to a Rayleigh distribution, and we obtain the ergodic channel capacity in each case. For a channel represented by a random matrix $\mathbf{H}$, the channel capacity is also random and we must obtain the capacity through an iterative process. We work with the Monte Carlo methods considering a thousand iterations to estimate the capacity in every case. 
To get the final ergodic channel capacity, we obtain the arithmetic average of these thousand individual capacities. Also, we introduce numerical results to quantify the influence that the correlation matrix $\mathbf{R}_{\mathbf{x x}}$ has on capacity by analyzing scenarios, according to the power allocation strategy employed.

\section{Channel capacity using I-AST}

We obtained the mathematical model and the channel capacity expression for the I-AST and analyze how its channel capacity improves compared to a traditional MIMO system (TMS). For the TMS, the correlation matrix will be considered as an identity matrix (unless specified differently), which means that equal power is allocated to each and every transmit antenna employed, and that all signals are uncorrelated. The ergodic channel capacity for TMS, where there is no antenna selection, is defined by:

$$
\bar{C}=\varepsilon\left[\max _{\substack{T_{r}(\mathbf{R} \mathbf{x x}) \\=M_{T}}} \log _{2}\left(\left|\mathbf{I}_{M_{R}}+\frac{E_{s}}{M_{T} N_{0}} \mathbf{H R}_{\mathbf{x x}} \mathbf{H}^{H}\right|\right)\right] .
$$

Likewise, the ergodic channel capacity using I-AST is defined as:

$$
\bar{C}=\varepsilon\left[\max _{K, \mathbf{R}_{\overline{\mathbf{x}}}} \log _{2}\left(\left|\mathbf{I}_{M_{R}}+\frac{E_{s}}{P N_{0}} \mathbf{H}_{K} \mathbf{R}_{\overline{\mathbf{x x}}} \mathbf{H}_{K}^{H}\right|\right)\right] .
$$

A comparison is shown in Figure 3, where channel capacity is shown as a function of the signal-to-noise ratio (SNR), for the four different antenna configurations. Figure 3 shows clearly the advantage of using I-AST by an increase in ergodic capacity for the $4 \times 1,4 \times 2$, and $4 \times 3$ cases, for all the SNR values considered. This technique improves the channel capacity in comparison to that of TMS, except for the case of full rank $(4 \times 4$ array), where the channel capacity converges to the same value in both cases (TMS and I-AST), because there is no chance to

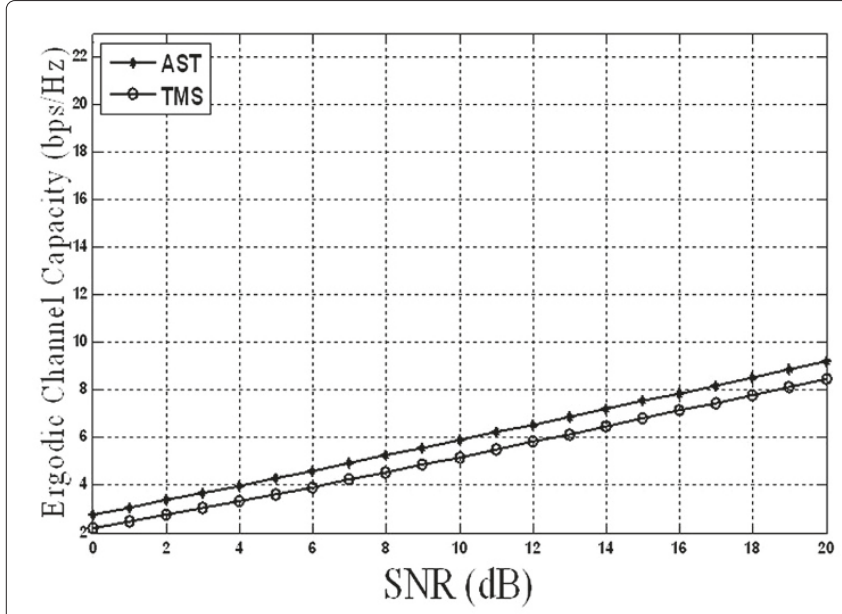

a

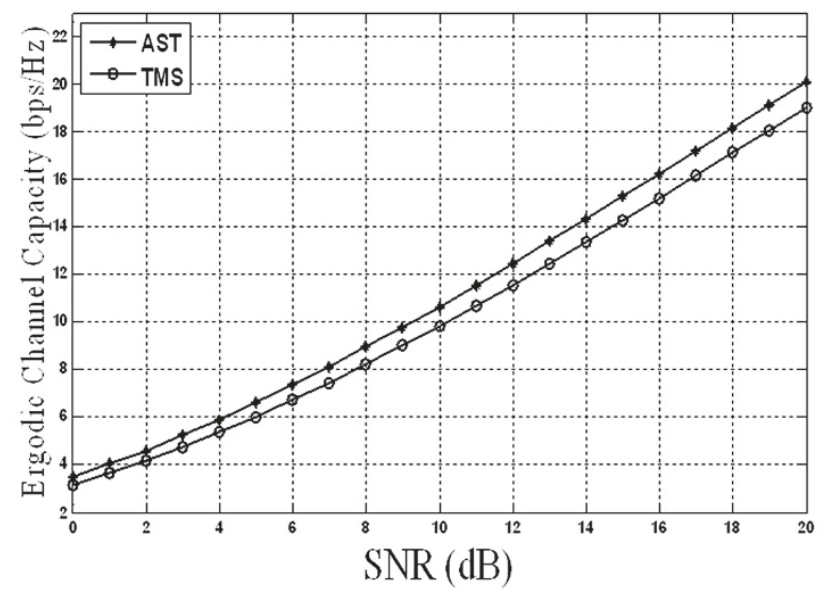

c

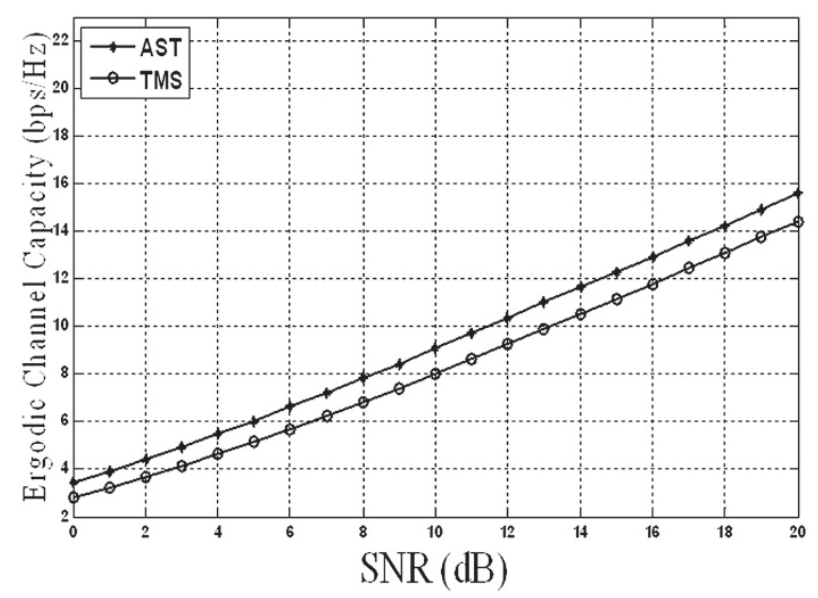

b

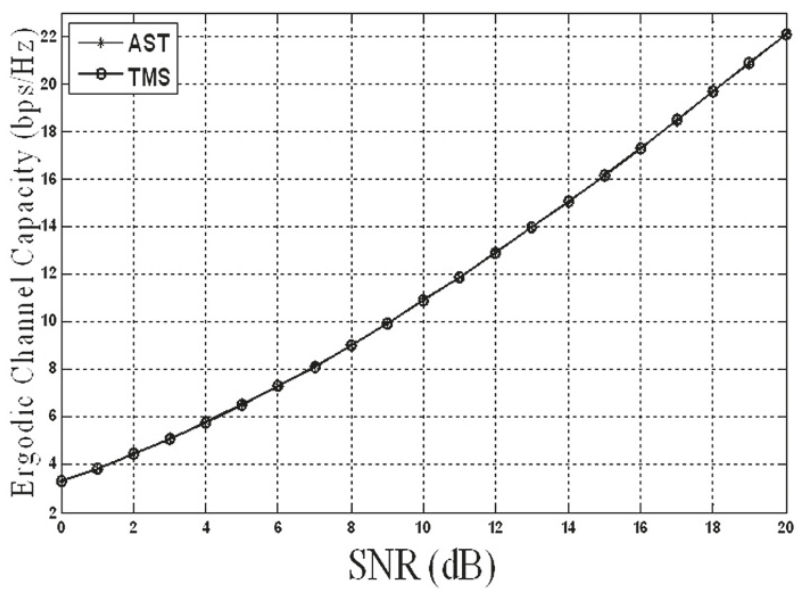

d

Figure 3 AST vs TMS. (a) $4 \times 1$, (b) $4 \times 2$, (c) $4 \times 3$, and (d) $4 \times 4$. Figure showing four plots of results for antenna selection vs traditional MIMO. 
select a different antenna set. For the cases of $4 \times 1,4 \times 2$, and $4 \times 3$, channel capacity using I-AST is better than that of TMS for all the values of SNR, because I-AST finds the set of antennas that represents the highest capacity, unlike TMS that just takes those available antennas according to the array employed. Since our purpose is to use the I-AST, it is not important which algorithm for AST is used since AST and I-AST converge to the same channel capacity, because both techniques work under the same principle of choosing those antennas that provide the highest capacity, but through different algorithms. Note that ergodic capacity increases as the number of transmit antennas increases.

The next step is to incorporate a power allocation technique to improve channel capacity. We show these results in the following subsections.

\section{Channel capacity using WPA}

The ergodic channel capacity using WPA is defined as:

$$
\bar{C}=\varepsilon\left[\max _{\sum_{i=1}^{r} \gamma_{i}=M_{T}} \sum_{i=1}^{r} \log _{2}\left(1+\frac{E_{s} \gamma_{i}}{M_{T} N_{0}} \lambda_{i}\right)\right] .
$$

Now, in Figure 4, we show the comparison between TMS and WPA under the same four-antenna configurations. The improvement of the channel capacity using WPA is clearly evident for the $4 \times 4$ array and is distinguishable for the complete range of the SNR employed. For the $4 \times 3$ array, the advantage of using WPA is evident for an SNR level from 0 to $16 \mathrm{~dB}$. In the case of a $4 \times 2$ array, the advantage of using WPA is minimum and just distinguishable at low SNR (from 0 to $8 \mathrm{~dB}$ ). Finally, there is no difference for the $4 \times 1$ array, because there is no power allocation strategy to perform, since there is only one transmitting antenna.

\section{Influence of correlation matrix}

It is well known that the correlation matrix can change mainly according to the power allocation. Therefore, we want to analyze the channel capacity depending on the variations of the correlation matrix.

To develop completely Equation 15, we have to get an expression for the correlation matrix. In this way, we can obtain $\mathbf{R}_{\overline{\mathbf{x x}}}$ using the model of the correlation matrix developed by [16] and used subsequently by [17], which is given by:

$$
\mathbf{R}_{\overline{\mathbf{x x}}}(\alpha)=\alpha \mathbf{I}+(1-\alpha) \mathbf{u u}^{H},
$$

where $\alpha$ is a real number that satisfies $0 \leq \alpha \leq \frac{P}{P-1}$, I is the identity matrix, and $\mathbf{u}$ is a vector of size $P \times 1$ and is defined as $\mathbf{u}=\left[\begin{array}{llll}1 & 1 & \ldots & 1\end{array}\right]^{T}$.

The expression for the ergodic channel capacity, using I-AST and taking into account the influence of the correlation matrix, is defined by:

$$
\bar{C}=\varepsilon\left[\max _{K, \mathbf{R}_{\overline{\mathbf{x}}}} \log _{2}\left(\left|\mathbf{I}_{M_{R}}+\frac{E_{s}}{P N_{0}} \mathbf{H}_{K} \mathbf{R}_{\overline{\mathbf{x x}}}(\alpha) \mathbf{H}_{K}^{H}\right|\right)\right],
$$

which gets its minimum value when the covariance matrix has all its elements set up to one. This means that all the channel coefficients are correlated. We denote this matrix
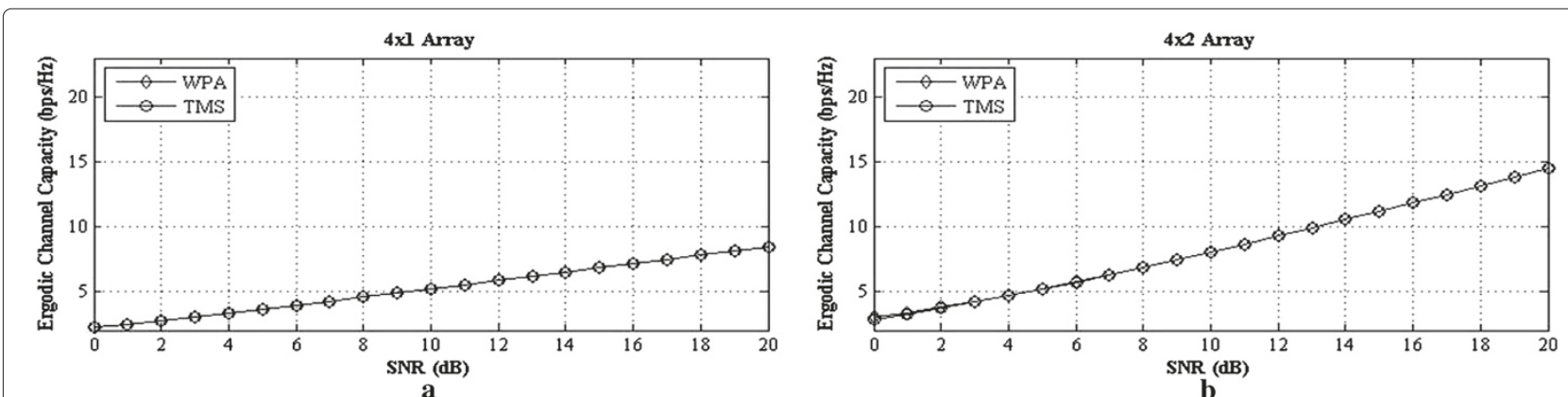

$4 \times 3$ Array
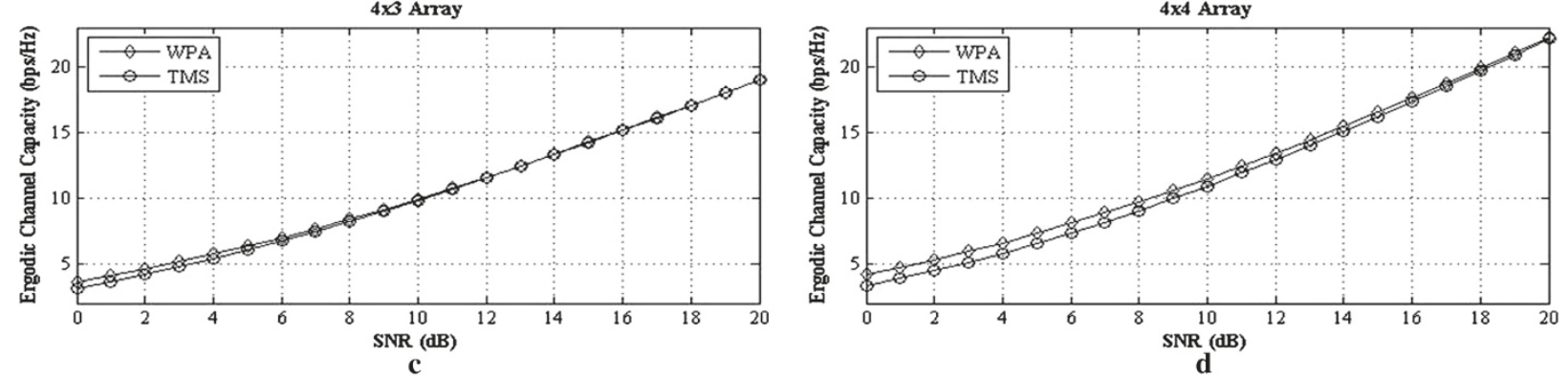

Figure 4 WPA vs TMS. (a) $4 \times 1$, (b) $4 \times 2$, (c) $4 \times 3$, and (d) $4 \times 4$. Figure showing four plots of results for water pouring vs traditional MIMO. 


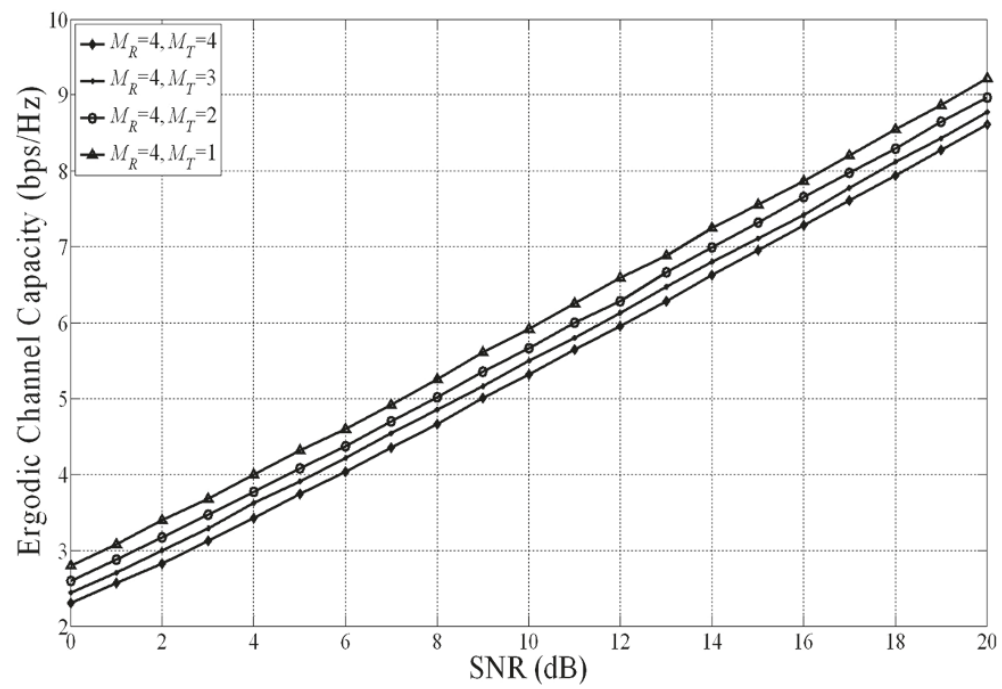

Figure 5 Channel capacity using I-AST with $\mathbf{R}_{\overline{\mathbf{x x}}}(\mathbf{0})$. Figure showing capacity of incremental AST with matrix of all ones as correlation matrix.

as $\mathbf{R}_{\overline{\mathbf{x x}}}(0)$, i.e., $\alpha=0$ in Equation 17. Thus, the minimum ergodic capacity in (18) is given by:

$$
\bar{C}_{\min }=\left.\bar{C}\right|_{\mathbf{R}_{\overline{\mathbf{x x}}}(0)} \text {. }
$$

Figure 5 shows the ergodic channel capacity for I-AST when the covariance matrix is $\mathbf{R}_{\overline{\mathbf{x x}}}(0)$, for the four different antenna configurations. Unlike the other typical cases, we can see that channel capacity decreases according to incremental number of transmit antennas, due to the high correlation provided by matrix $\mathbf{R}_{\overline{\mathbf{x x}}}(0)$, which represents a negative influence over the channel capacity. This means that a $4 \times 1$ array shows higher channel capacity than that of a $4 \times 2$ array. In the same way, a $4 \times 2$ array presents higher capacity than that of a $4 \times 3$ array, and finally, a $4 \times 3$ array also shows higher capacity than that of a $4 \times 4$ array. Likewise, the ergodic channel capacity gets its maximum with $\mathbf{R}_{\mathbf{x} \mathbf{x}}(1)$, i.e., $\alpha=1$ in Equation 17, which is an identity matrix. This means that there is no correlation between the transmitted signals, and equal power is allocated to

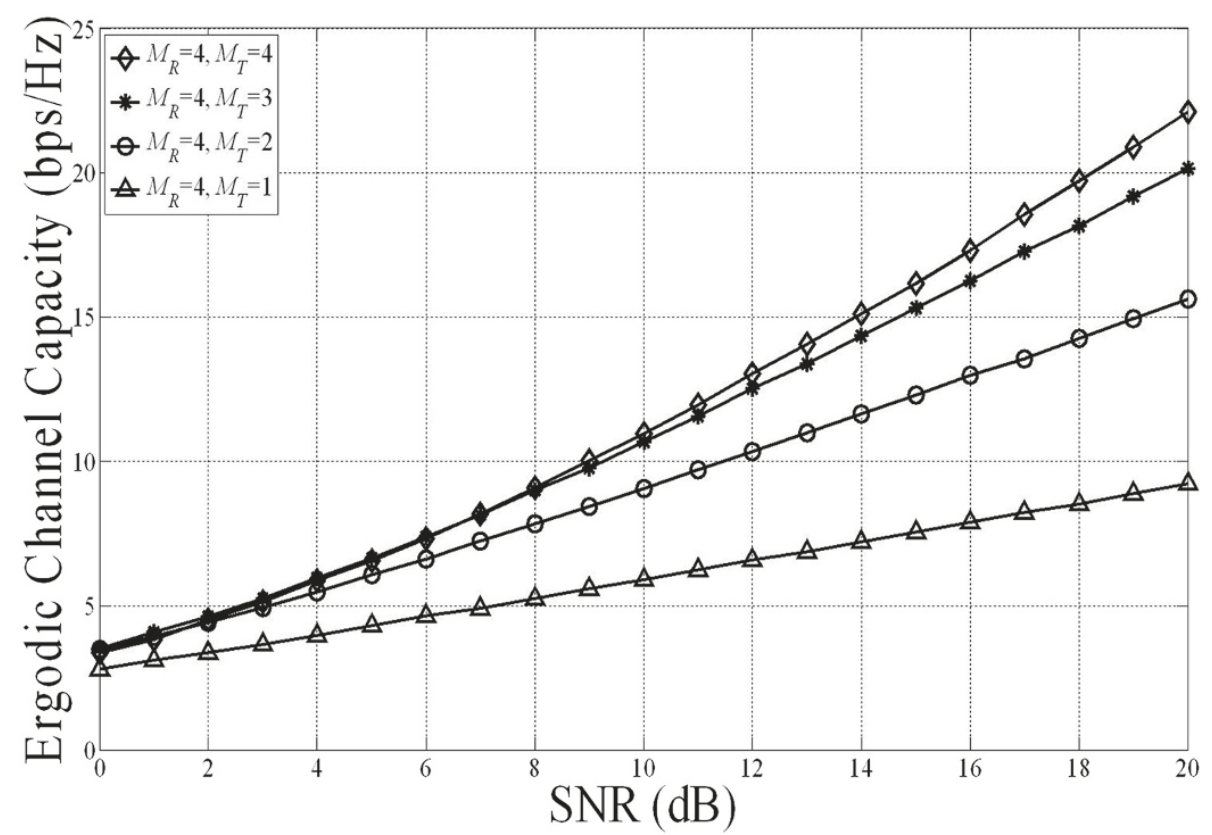

Figure 6 Channel capacity using I-AST with $\mathbf{R}_{\overline{\mathbf{x x}}}(\mathbf{1})$. Figure showing capacity of incremental AST with identity matrix as correlation matrix. 


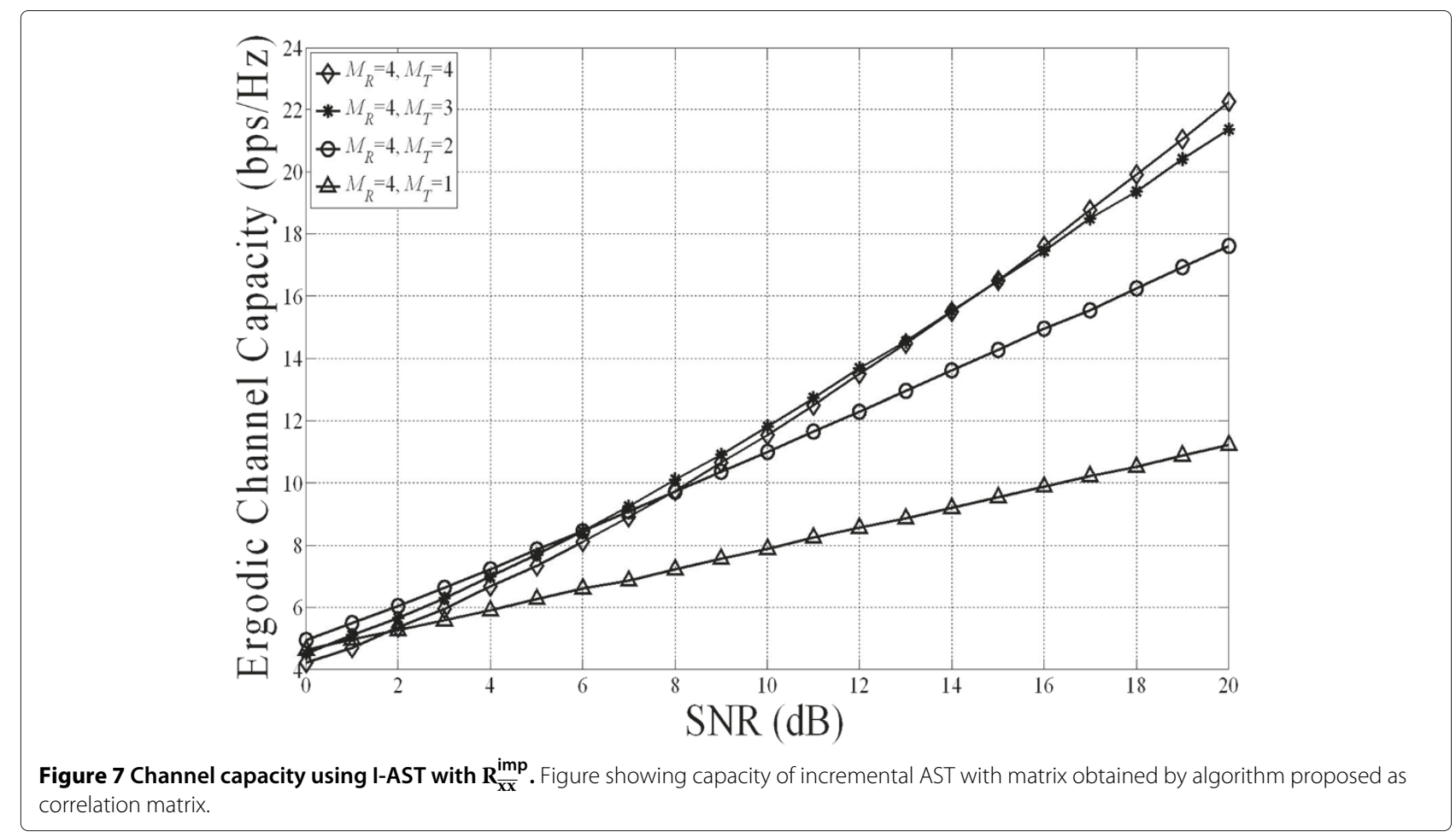

each antenna. This channel capacity is described by:

$$
\bar{C}_{\max }=\left.\bar{C}\right|_{\mathbf{R}_{\overline{x x}}(1)} \text {. }
$$

Figure 6 shows how the ergodic channel capacity with $\mathbf{R}_{\overline{\mathbf{x}} \mathbf{x}}(1)$ varies with respect to the SNR. In this figure, we can see that capacity increases according to the incremental number of transmit antennas. It is clear that at high SNR (from 7 to $20 \mathrm{~dB}$ ), the channel capacity increases with the incremental number of antennas. We can see the same behavior at low SNR, but the channel capacity of the $4 \times 3$

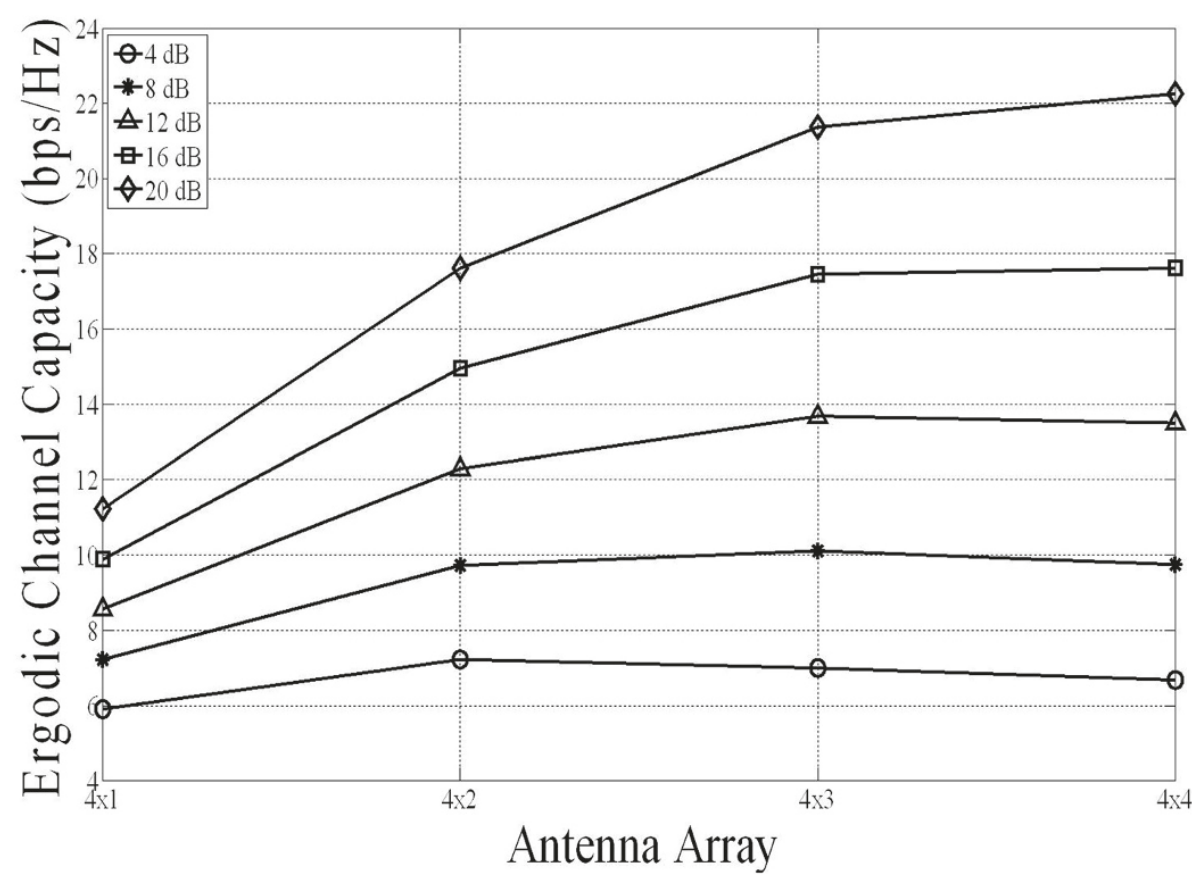

Figure 8 Ergodic channel capacity vs the number of transmit antennas. Figure showing capacity as the number of antennas is varied. 
array is somewhat higher than that of a $4 \times 4$ array, from 0 to $6 \mathrm{~dB}$. This is due to the effect of selecting the best subset of three antennas. For the system of $4 \times 4$ antennas, we are not performing any antenna selection; therefore, one of those channels produces a negative contribution to the capacity, which makes the channel capacity of the $4 \times 3$ case better at low SNR.

\section{Channel capacity of the proposed model}

Our model uses I-AST to select the better set of antennas and then allocates power by carrying out WPA. Recall that the ergodic channel capacity expression for this proposed model is given by Equation 14. Figure 7 shows how the channel capacity of our model varies according to the SNR. We can see that all the results shown in Figure 7 are better than their counterpart shown in Figure 6. Therefore, the use of I-AST and WPA improves channel capacity. From Figure 7, we can see that at high SNR (from 15 to $20 \mathrm{~dB}$ ) the channel capacity increases with the incremental number of transmit antennas. This statement changes for the other range of SNR. For instance, in the range of 7 to $14 \mathrm{~dB}$, the best channel capacity is obtained for the
$4 \times 3$ array, and in the range of 0 to $6 \mathrm{~dB}$, the best channel capacity is obtained for the $4 \times 2$ array. The higher channel capacity at low SNR, for the $4 \times 3$ and $4 \times 2$ arrays, is due to the advantage of using I-AST and WPA, since the selection of the best set of $P$ transmit antennas working together with the optimal strategy of power allocation, can provide a higher channel capacity.

This behavior is much easier to visualize if we plot the channel capacity versus the number of transmit antennas. In Figure 8, we show the ergodic channel capacity as a function of the number of transmit antennas, for different values of SNR. In this figure, we can see that at $4 \mathrm{~dB}$ of SNR, the capacity obtained with a $4 \times 2$ array is better than that of a $4 \times 3$ array and that of a $4 \times 4$ array. At 8 and 12 $\mathrm{dB}$, the capacity obtained with a $4 \times 3$ array is better than that of a $4 \times 4$ array. Finally, at 16 and $20 \mathrm{~dB}$, the capacity obtained with a $4 \times 4$ array is better than all the antenna configurations.

Figure 8 also helps us to determine how many antennas and which SNR level we need to obtain for a specific channel capacity. For instance, if we need $10 \mathrm{bps} / \mathrm{Hz}$, we can use a $4 \times 1$ array at $16 \mathrm{~dB}$ or a $4 \times 2$ array at $12 \mathrm{~dB}$

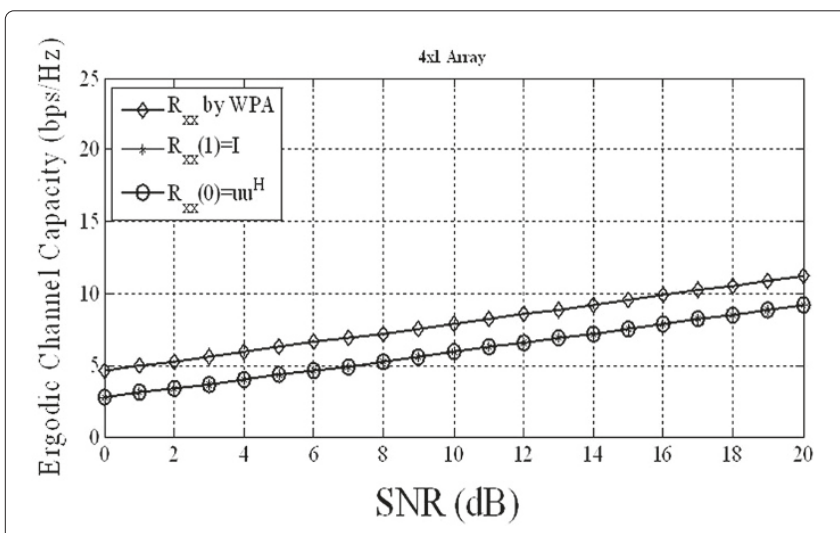

a

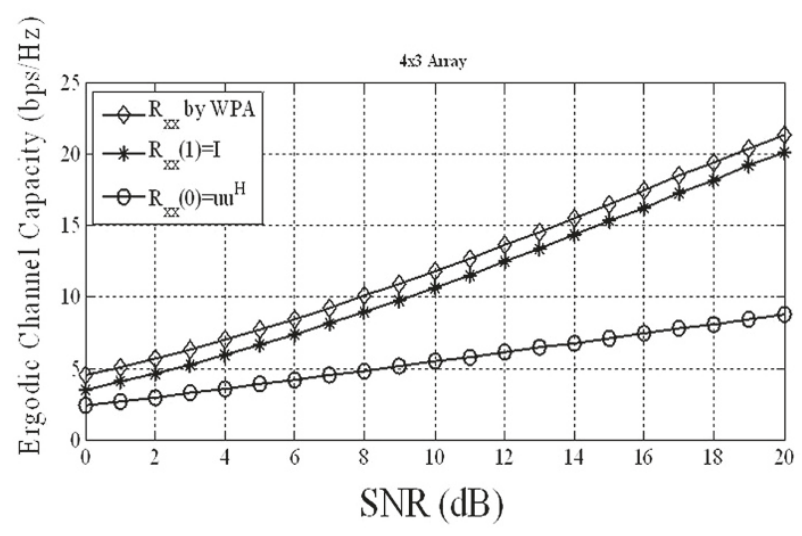

c

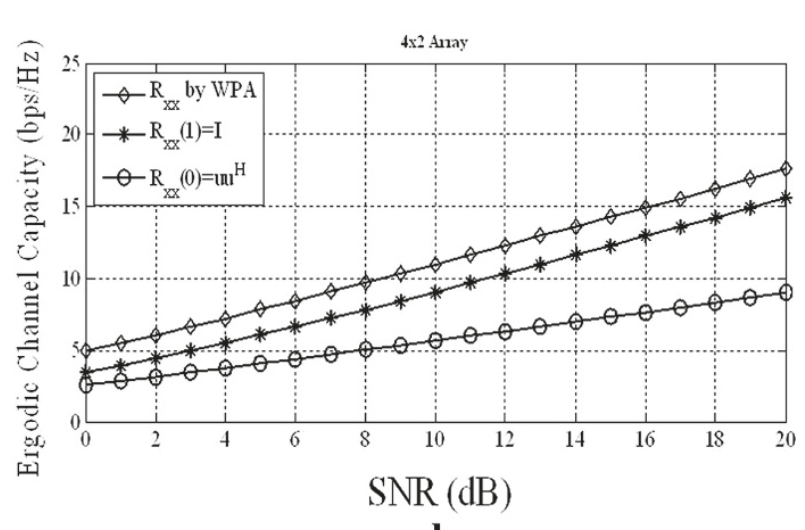

b

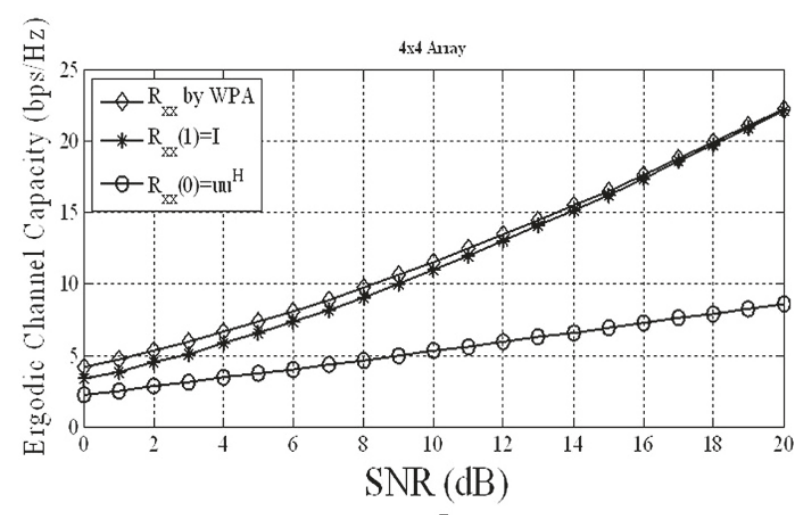

d

Figure 9 Comparison of the channel capacity using the three different methods. (a) $4 \times 1$, (b) $4 \times 2$, (c) $4 \times 3$, and (d) $4 \times 4$. Figure showing four plots of capacity as the number of antennas is varied. 
or a $4 \times 3$ array at $8 \mathrm{~dB}$. In this way, we obtain the same channel capacity but at different SNR levels $(16,12$, or 8 $\mathrm{dB}$, respectively) or with different antenna configurations $(4 \times 1,4 \times 2$, or $4 \times 3$, respectively). In case that we need $17 \mathrm{bps} / \mathrm{Hz}$, we can use a $4 \times 2$ array at $20 \mathrm{~dB}$ or $4 \times 3$ array at $16 \mathrm{~dB}$, and we obtain the same channel capacity but at different SNR levels (20 or $16 \mathrm{~dB}$, respectively) or with different antenna configurations $(4 \times 2$ or $4 \times 3$, respectively).

\section{Lower and upper bounds}

So far, we have seen three different methods that use IAST to obtain a better channel capacity. These methods change each other according to the correlation matrix, these methods were indicated as:

- I-AST with $\mathbf{R}_{\overline{\mathbf{x x}}}(0)$

- I-AST with $\mathbf{R}_{\overline{\mathbf{x x}}}(1)$

- I-AST with $\mathbf{R}_{\overline{\mathbf{x x}}}$ obtained by WPA $\left(\mathbf{R}_{\overline{\mathbf{x}} \mathbf{x}}=\mathbf{R}_{\overline{\mathbf{x}} \mathbf{i}}^{\mathrm{imp}}\right)$

Now, we can compare these three methods to see how the ergodic channel capacity varies in each one. Figure 9 shows the comparison between these three methods for the four-antenna configurations studied before. This figure clearly shows that channel capacity gets its maximal value (in the four-antenna configurations employed) when $\mathbf{R}_{\overline{\mathbf{x}} \mathbf{x}}$ is obtained by WPA $\left(\mathbf{R}_{\overline{\mathbf{x x}}}^{\mathrm{imp}}\right)$. Likewise, the channel capacity gets its minimal value for $\mathbf{R}_{\overline{\mathbf{X X}}}(0)$.
Therefore, we can establish lower and upper bounds for the channel capacity formulation of the model proposed here as follows:

$$
\bar{C}_{\min } \leq \bar{C}_{\max } \leq \bar{C}_{\mathrm{imp}}
$$

where $\bar{C}_{\min }, \bar{C}_{\max }$, and $\bar{C}_{\mathrm{imp}}$ are given by Equations 14,19 , and 20 , respectively.

In other words, we can say that a lower bound is obtained for $\mathbf{R}_{\overline{\mathbf{x}} \mathbf{x}}(0)$, a middle value for $\mathbf{R}_{\overline{\mathbf{x x}}}(1)$, and an upper bound with $\mathbf{R}_{\overline{\mathbf{x x}} \mathbf{p}}^{\mathrm{imp}}$. These are bounds according to the influence of the correlation matrix and the variations of parameter $\alpha$ in our formulation.

So far, we have only worked with cases up to $4 \times 4$, but results will change if we increase the number of antennas. The next scenario that we include is an array of $8 \times 8$ antennas as a full rank, and we vary the number of transmitting antennas from 8 to 1 . We show only the case of I-AST with $\mathbf{R}_{\overline{\mathbf{x x}}}$ obtained by WPA $\left(\mathbf{R}_{\overline{\mathbf{x x}}}^{\mathrm{imp}}\right)$, since it was the approach with the highest channel capacity for the cases analyzed previously. Figure 10 shows how the channel capacity increases according to the incremental number of transmit and receive antennas, reaching twice the channel capacity approximately, which shows that the proposed model is scalable to bigger MIMO configurations.

\section{Conclusions}

One of the main challenges of wireless communications systems is to improve the channel capacity. For this

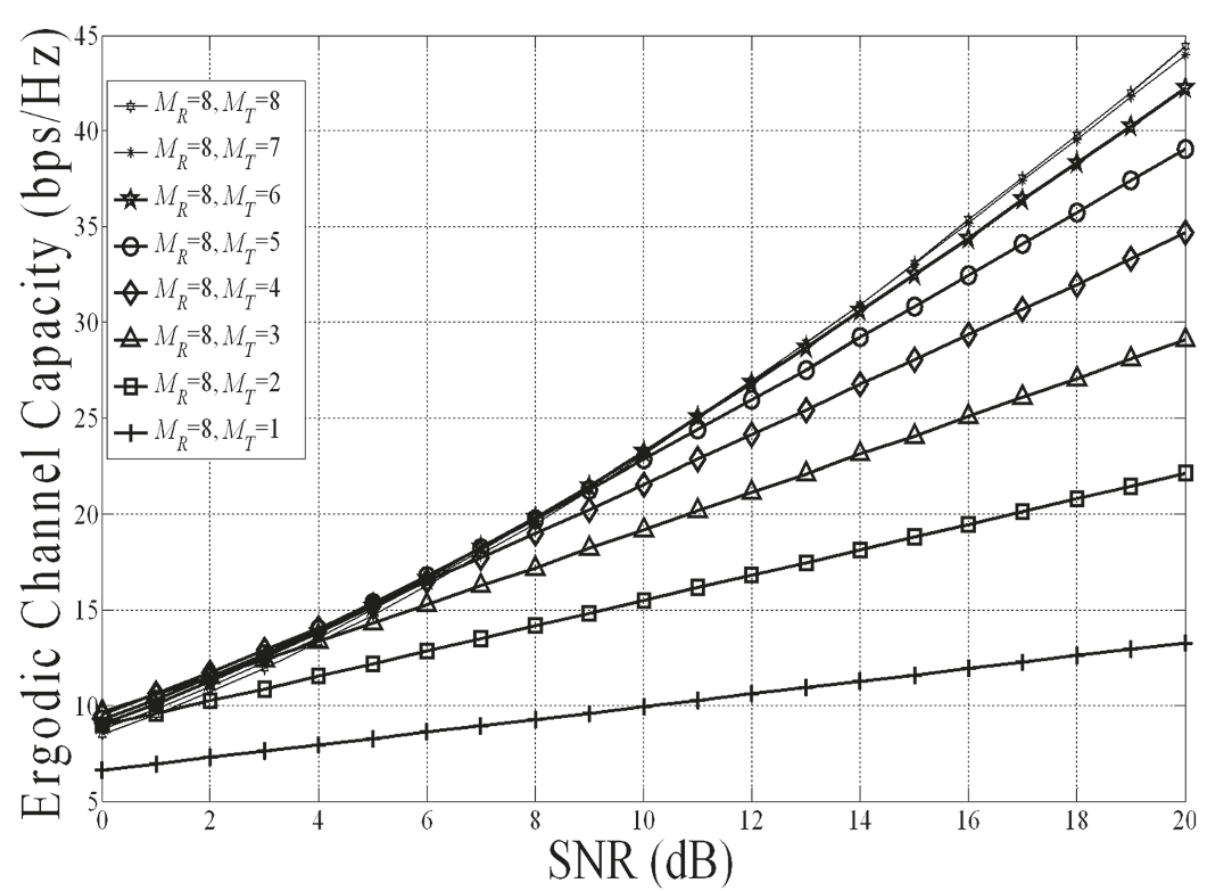

Figure 10 Channel capacity using I-AST with $\mathbf{R}_{\overline{\mathbf{x x}}}^{\mathrm{imp}}$. Figure showing plot of capacity with correlation matrix. 
reason, we have analyzed and used different techniques to face and overcome this challenge. We have employed I-AST, because this technique helps us to choose those antennas linked with the channels that provide better transmit conditions, as well as to determine the channel state information. On the other hand, having knowledge of the channel state, we are able to apply the WPA and allocate power in an efficient way under the rule of assigning more power to the channel that has better conditions. Employing these two techniques (I-AST and WPA), we have shown that the channel capacity was significantly improved, with higher capacity values than those obtained using traditional systems which just spread equal power among all the transmit antennas.

Using both techniques, we also obtain a more costefficient MIMO communication system; because AST reduces the cost and the number of the RF chains employed, and WPA allocates the power efficiently. This contributes, in certain manner, to work towards the new Green Wireless Technology, helping in this way, to the conservation of the environment and the natural resources by saving or using more efficiently the transmission energy. As a future work of interest is the integration of channel estimation algorithms with I-AST and WPA.

\section{Competing interests}

The authors declare that they have no competing interests.

\section{Acknowledgements}

The authors would like to thank the Telecommunications Focus Group and the RFID Research Chair on Electronics and Telecommunications at Tecnologico de Monterrey for its financial support for the development of the techniques presented in this manuscript.

Received: 26 December 2013 Accepted: 16 December 2014

Published: 30 December 2014

\section{References}

1. RN A Paulraj, D Gore, Introduction to Space-time Wireless Communications. (Cambridge University Press, USA, 2005)

2. MA Beach, DP McNamara, PN Fletcher, P Karlsson, in Proceedings of the Eleventh International Conference on Antennas and Propagation. MIMO - a solution for advanced wireless access? (London, UK, 2001), pp. 231-235

3. M Jankiraman, Space-time Codes and MIMO Systems. (Artech House, Boston, 2004)

4. S Sanayei, A Nosratinia, Antenna selection in MIMO systems. IEEE Commun. Mag. 42, 68-73 (2004)

5. Q Ma, C Tepedelenlioglu, Antenna selection for space-time coded systems with imperfect channel estimation. IEEE Trans. Wireless Commun. 6, 710-719 (2005)

6. AB Narasimhamurthy, C Tepedelenlioglu, Antenna selection for MIMO-OFDM systems with channel estimation error. IEEE Trans. Vehicular Technol. 58, 2269-2278 (2009)

7. S Sandhu, RU Nabar, DA Gore, A Paulraj, in Conference Record of the Thirty-Fourth Asilomar Conference on Signals, Systems and Computers. Near-optimal selection of transmit antennas for a MIMO channel based on Shannon capacity (Pacific Grove California, USA, 2000), pp. 567-571

8. R Yellapantula, Y Yingwei, R Ansari, Antenna selection and power control in $\mathrm{MIMO}$ systems with continuously varying channels. IEEE Commun. Lett. 13, 480-482 (2009)

9. RW Heath Jr, DJ Love, Multimode antenna selection for spatial multiplexing systems with linear receivers. IEEE Trans. Signal Process. 53, 3042-3056 (2005)
10. G Zhaozhi, L Sheng, in Proceedings of the IEEE International Conference on Computer, Mechatronics, Control and Electronic Engineering (CMCE). Effect of antenna selection on channel capacity of MIMO system (IEEE Changchun, China, 2010), pp. 471-473

11. NB Mehta, S Kashyap, AF Molisch, Antenna selection in LTE: from motivation to specification. IEEE Commun. Mag. 50, 144-150 (2012)

12. GXiang, O Edfors, L Jianan, F Tufvesson, in Proceedings of the IEEE Global Communications Conference (GLOBECOM). Antenna selection in measured massive MIMO channels using convex optimization (IEEE Atlanta, GA, USA, 2013), pp. 129-134

13. AF Molisch, Y Choi, Capacity of MIMO systems with antenna selection. IEEE Trans. Wireless Commun. 6, 1759-1772 (2005)

14. M Gharavi-Alkhansari, AB Gershman, in in Space-time Processing for MIMO Communications, ed. by Gershman A, Sidiropoulos N. Antenna subset selection in MIMO communication systems (John Wiley \& Sons Ltd England, 2005), p. 245

15. TM Cover, JA Thomas, Elements of Information Theory. (John Wiley \& Sons Ltd, USA, 2006)

16. PJ Voltz, Characterization of the optimum transmitter correlation matrix for MIMO with antenna subset selection. IEEE Trans. Commun. 51, 1779-1782 (2003)

17. NB Mehta, AF Molisch, in MIMO Systems Technology for Wireless Communications, ed. by G Tsoulos. Antenna selection in MIMO systems (John Wiley \& Sons Ltd USA, 2006)

\section{doi:10.1186/1687-1499-2014-228}

Cite this article as: Cuan-Cortes et al:: MIMO channel capacity using antenna selection and water pouring. EURASIP Journal on Wireless Communications and Networking 2014 2014:228.

\section{Submit your manuscript to a SpringerOpen ${ }^{\odot}$ journal and benefit from:}

- Convenient online submission

Rigorous peer review

- Immediate publication on acceptance

- Open access: articles freely available online

- High visibility within the field

- Retaining the copyright to your article

Submit your next manuscript at $>$ springeropen.com 